\title{
Low molecular weight gut polyphenols metabolites and its nutritional relevance as effectors for attenuating neuroinflammation
}

\author{
Diogo Carregosa ${ }^{1,2}$, Rafael Carecho ${ }^{1,3}$, Inês Figueira ${ }^{2,3}$ and Cláudia Nunes dos Santos ${ }^{1,2}$ \\ ${ }^{1}$ CEDOC, NOVA Medical School|Faculdade de Ciências Médicas, Universidade NOVA de Lisboa, Campo dos \\ Mártires da Pátria, 130, 1169-056, Lisboa, Portugal, \\ ${ }^{2}$ iBET, Instituto de Biologia Experimental e Tecnológica, Av. da República, Apartado 12, 2781-901, Oeiras, Portugal \\ and \\ ${ }^{3}$ Instituto de Tecnologia Química e Biológica António Xavier, Universidade Nova de Lisboa, Av. da República, \\ 2780-157, Oeiras, Portugal
}

\begin{abstract}
The burden of neurodegenerative disorders has been increasing as a consequence of a growing elderly population. These multifactorial diseases do not have a cure or efficient treatments. Due to its mechanistic complexity, prevention and treatment will require novel multi-targeted therapeutic strategies, targeting different disease hallmarks. The possibility of altering the progression and development of diseases through diet is an emerging as attractive approach with increasing supporting data. Epidemiological and clinical studies have highlighted the health potential of diets rich in fruits and vegetables. Studies with dietary (poly)phenols have been showing their multipotent and pleiotropic ability to modulate several cellular and molecular pathways and in that sense, dietary (poly)phenols can emerge as an alternative, with potential to be further explored. However, the precise contribution of dietary (poly)phenols and circulating (poly)phenol metabolites to human health is still in the beginning of being elucidated. Absorption and blood concentrations of some (poly)phenols is quite low, which can hamper the research in terms of understanding their effects in specific biomarkers of disease.

The difficulty in demonstrating (poly)phenols true effects can also be justified by the uncertain metabolic fate that dietary (poly) phenols can have. In fact, it is necessary to identify the bioavailable metabolites resulting from (poly)phenol ingestion through the diet, as well as their ability to overcome and/or interact with cellular barriers and reach target tissues, in this case reach the brain. Having this in mind, it will be reviewed the current knowledge on the molecular mechanisms underlying (poly)phenol metabolites effects and their role on neuroinflammation one central hallmark, common in all neurodegenerative diseases.
\end{abstract}

\section{Acknowledgements}

this work has received funding from European Research council (ERC) under the European Union's Horizon 2020 research and innovation programme under grant agreement No 804229

\section{Conflict of Interest}

There is no conflict of interest 